\title{
Network Topological Modeling on Private Equity Investment
}

\author{
Tian Lv and Jinchuan $\mathrm{Ke}^{*}$ \\ Beijing Jiaotong University, Beijing 100044, P.R.China \\ ${ }^{*}$ Corresponding author
}

\begin{abstract}
A private equity investment network can be composed of nodes and edges which represent the regions and the private equity relationship between regions respectively. This paper analyzes the network's density, average path length, clustering coefficient degree, betweeness, closeness centrality and its coreperiphery structure to investigate the characteristics of the crossregional PE investment network. The result indicates that China's PE investment varies from regions; whether they are in the core area or periphery area, enterprises are more likely to invest in or be invested by enterprises in the core area.
\end{abstract}

Keywords-private equity investment; complex network; regional difference

\section{RESEARCH OVERVIEW}

Complex network is a kind of system structure formed by the connection between the nodes, which describe the intrinsic composition of complex systems and their interaction with each other on a network, and then analyzes the relationship between the intrinsic structure and the overall properties of complex systems (BarabásiA-L, etc. 1999). In complex networks, a node represents an individual or an organization, and a connection edge represents an association between individuals or organizations (Albert, etc. 1999). For example, Ming Zheng etc. (2011) constructed a PE network, and investigated the structural characteristics.

Different regions' companies invest with PE in each other, which forms a complex social network whose nodes represent the regions and its connection edges represent the investment relationships. This paper studies the regional characteristics of $\mathrm{PE}$ investment in China through the topological structure analysis, centrality analysis and core periphery structure analysis under PE investment weighted network.

\section{RESEARCH METHODS}

This paper constructs a cross-regional complex private equity investment network with the private equity relationships among enterprises in different regions.

Nodes: each node in the network represents a region.

Edge: the edge of the network represents the private equity relationship between enterprises.

The rules of connecting: if there is a cross-regional private equity relationship between two different regions' enterprises, then connect the two nodes, and ignore the private equity relationships within a region. The cross-regional private equity investment network is directional, if region A's enterprises invested region B's enterprises, then the direction of the edge point from the node which represents region $\mathrm{A}$ to the node that represents region $B$.

According to above modeling strategy, cross-regional private equity relationship network based on the capital market can be described by whether or not there is a cross-regional private equity investment activity between two regions, which can be expressed as a mathematical expression $(\mathrm{F}, \mathrm{A})$.

Where $\mathrm{F}$ is defined as a collection of $\mathrm{m}$ regions, each $F_{i}$ represents a network node;

$\mathrm{A}$ is an adjacency matrix $(\mathrm{m} \times \mathrm{m})$ of the cross-regional private equity relationship between regions. The adjacency matrix is a matrix consisting of 0 and 1 , that is, if there is a cross-regional private equity investment activity between region $\mathrm{i}$ and region $\mathrm{j}$, then $\mathrm{A}_{\mathrm{ij}}=1$, and that in the network graph, the two nodes representing regions are adjacent; otherwise, $\mathrm{A}_{\mathrm{ij}}=0$, the two nodes are not adjacent.

The weight of edge: weight the edge by the amount of cross-regional private equity investment between two nodes.

The weighted network can be described by the set $\mathrm{G}=(\mathrm{N}$, $\mathrm{W})$, where $\mathrm{N}$ is the number of the region; $\mathrm{W}$ is the weighted adjacency matrix network, the matrix element $w_{i j}\left(w_{i j} \geq 0\right)$ represents the weight of the edge between two nodes which are connected by cross-regional private equity relationships. Here, $\mathrm{w}_{\mathrm{ij}}$ represents the investment amount of the enterprises subordinate to region $i$ that invested enterprises subordinate to region $\mathrm{j}$ in a period of time. If the PE investment amount is larger, the weight of the edge is greater, too; On the other hand, the weight of the edge is smaller. In the practical sense, the greater the PE investment amount in two regions, the more the relationship between the two, the closer the economic ties.

\section{A. Network Topological Structure Analysis}

In the network, we can use the average path length and clustering coefficients to analyze the structural characteristics of the network. If a network has a small average path length and a large clustering coefficient, it is considered to have "small world effect".

$$
L^{-1}=\frac{1}{\frac{1}{2} N(N+N)} \sum_{i \geq j} d_{i j}{ }^{-1}
$$

The clustering coefficient is used to characterize the small group form of the network. The formula is as follows: 


$$
C=\frac{1}{n} \sum_{i} C_{i}
$$

In which, $\mathrm{C}_{\mathrm{i}}$ represent the local clustering coefficient of node $i, i=1,2, \ldots, n$.

\section{B. Centrality Analysis}

The centrality analysis includes degree analysis, betweenness analysis and closeness centrality analysis.

1) Degree: Degree measures the number of other nodes connected to the node. In the weighted network, the node degree of the formula is as follows:

$$
C_{i}^{A}=\sum_{j \neq i}^{n} w_{i j}
$$

Where: $w_{i j}$ is the edge weight between nodes $i$ and $j$.

2) Betweenness: The main idea of betweenness is: the more the number of nodes in the network is between the other nodes, the more the center is in the center, that is, many of the shortest paths between nodes have passed through this node. The calculation of the following formula is as follows:

$$
C_{i}^{B}=\frac{1}{(N-1)(N-2)} \sum_{j \in G, j \neq i} \sum_{k \neq i, k \neq j} \frac{n_{j k}(i)}{n_{j k}}
$$

Where:

$\mathrm{n}_{\mathrm{jk}}$ is the number of shortest paths that exist between nodes $\mathrm{j}$ and $\mathrm{k}$, representing the number of paths containing node $\mathrm{i}$ in the shortest path that exists between nodes $\mathrm{j}$ and $\mathrm{k}$.

3) Closeness centrality:The Closeness centrality is the reciprocal of the sum of the shortest paths of the node and all other nodes in the network, specifically expressed as:

$$
C_{i}^{C}=\frac{1}{\sum_{j=1}^{n} d_{i j}}
$$

\section{DATA}

This paper chooses cross-regional private equity investment activities as research the object, and do not consider private equity investment activities in the same region. Finally, 1066 private equity investment activities are selected as research objects from the Wind information database after excluding private equity investment activities whose investors and financiers are in the same region, non-domestic private equity investment activities, as well as incomplete data M\&A transactions, whose first disclosure date were between January 1, 2012- December 31, 2016.

According to the classification of the provincial administrative regions in China, the region of private equity investment is divided into 34 regions. We build private equity investment weighted network, and hope to get the regional characteristics of private equity investment during these 5 years by analyzing the topological structure, the centrality and the core-periphery structure with the help of the social network analysis software - UCINET.

\section{RESUlts AND ANALYSIS}

\section{A. Analysis of Network's Topological Structure}

The private equity relationship between the various regions is shown in Figure 1. The nodes represent the regions and there are 34 regions; the edges between the nodes represent the private equity relationships and there are 174 edges; the edges are directional and the arrows point to the financing side; the thickness of the edge represents the amount of private equity investment, which means the thicker the edge, the greater the amount of private equity investment. There are 34 nodes in this network, then the maximum number of edges between the 34 regions is $1122(34 \times 33)$, and the actual number of edges is 174 , so the network density is 0.1551 . This network density is relatively small, indicating that many nodes are not connected, so the network cohesion is low, which can also be seen from Figure 1 intuitive. Most nodes are in the periphery position, only connecting with three or four nodes, and the edges are very sparse; few nodes are in the core position, and only the edges between which and the nodes in the same core position are dense.

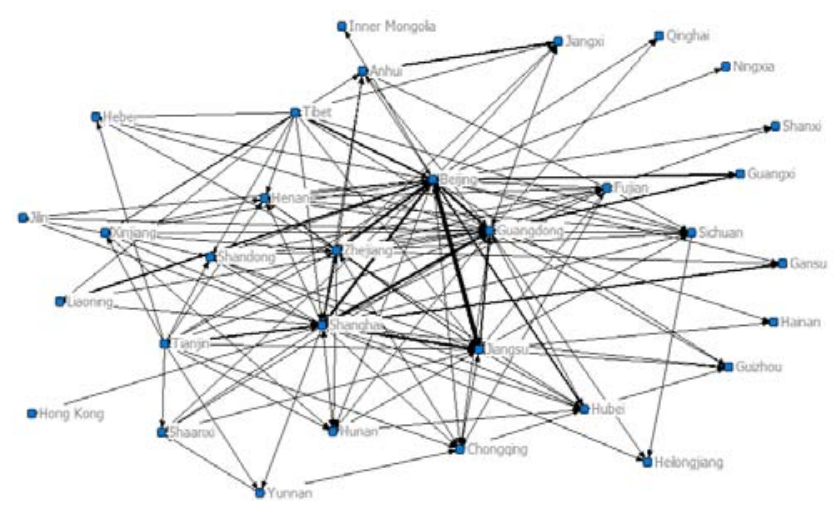

FIGURE I. CROSS-REGIONAL PEINVESTMENT NETWORK

Although the cohesion of the network is low, the average path length of this network is 1.790 , the clustering coefficient is 0.664 , which shows obvious small world effect. The average path length is the average shortest path of the whole network; longer average path length means more steps are needed for enterprises in a region to invest in or be invested by the enterprises in another region. The average path length of this network is 1.790 , indicating that two regions' enterprises can be linked through private equity relationships by less than two steps on average. The clustering coefficient can describe the small group form, the clustering coefficient of this network is 0.664 , which indicates that a small group with one or several nodes (Beijing / Shanghai / Guangdong / Jiangsu) has been formed. In this small group, there are many edges between nodes, so the network is highly interoperable.

The network's cohesion is very low and the network is relatively sparse, but it has shown a small world effect, indicating that China's private equity investment development is not even in different regions. Most regions do not have the 
value to be invested in private equity or the ability to invest in private equity, only some regions which connect with each other closely have this kind of value or ability, and forming a highly interoperable small group.

\section{B. Analysis of Network Centrality}

1) Degree: Degree reflects the mutual private equity relationships between various regions' enterprises. Degree is divided into out-degree and in-degree. Out-degree of a region describes how many regions (whose enterprises) that have been invested in PE by enterprises in this region, and in-degree of a region describes how many regions (whose enterprises) invested in PE in enterprises in this region. The greater the degree of a region, the more the number of regions connected to this region, the greater the influence of this region has, so the more is the region in the central position in the network.

TABLE I. THE LIST OF OUT-DEGREE \& INDEGREE IN PRIVATE EQUITY INVESTMENT COMPLEX NETWORK

\begin{tabular}{|c|c|c|c|c|}
\hline Order & Region & Out-degree & Region & In-degree \\
\hline 1 & Beijing & 2998091 & Beijing & 1824222 \\
\hline 2 & Shanghai & 2276147 & Jiangsu & 1548454 \\
\hline 3 & Guangdong & 1202022 & Guangdong & 1514931 \\
\hline 4 & Jiangsu & 786238 & Shanghai & 1012085 \\
\hline 5 & Zhejiang & 737807 & Zhejiang & 541662 \\
\hline 6 & Tianjin & 455293 & Hubei & 392460 \\
\hline 7 & Tibet & 451230 & Liaoning & 353052 \\
\hline 8 & Jiangxi & 225933 & Guangxi & 290200 \\
\hline 9 & Fujian & 186869 & Gansu & 277342 \\
\hline 10 & Hong Kong & 86800 & Anhui & 257484 \\
\hline \multicolumn{5}{|l|}{$\ldots$} \\
\hline 25 & Gansu & 0 & Xinjiang & 34895 \\
\hline 26 & Macao & 0 & Hainan & 32400 \\
\hline 27 & Henan & 0 & Qinghai & 29950 \\
\hline 28 & $\begin{array}{l}\text { Heilong- } \\
\text { jiang }\end{array}$ & 0 & Ningxia & 13663 \\
\hline 29 & Qinghai & 0 & Jiangxi & 13229 \\
\hline 30 & Liaoning & 0 & InnerMongolia & 5000 \\
\hline 31 & Jilin & 0 & Tibet & 0 \\
\hline 32 & Shaanxi & 0 & Hong Kong & 0 \\
\hline 33 & Hainan & 0 & Macao & 0 \\
\hline 34 & Taiwan & 0 & Taiwan & 0 \\
\hline
\end{tabular}

Table 1 shows out-degree and in-degree of various regions (weighted value). Due to the page limitation, only list the top 10 and last 10 regions. Beijing's out-degree and in-degree are ranked first, indicating that Beijing is in the central position of the whole network and has the greatest influence. Beijing is China's political center, enjoying the policy advantage, bringing together a lot of talent and money, so there is no doubt that it's the center of the network. In addition, the out-degree and in-degree of Shanghai, Guangdong, Jiangsu and Zhejiang provinces are also in the forefront, and much higher than that of other regions, which means these 4 regions are also in central position. Shanghai is China's economic center, whose financial system is the most developed in China; Shenzhen, located in Guangdong, is not only the location of the Shenzhen Stock Exchange, but also gathered a large number of small and medium-sized high-tech enterprises; besides, Jiangsu and Zhejiang are located in the southeast coast of China, whose economic resources are very rich. Therefore, these four regions have also become the main force of private equity investment.
There are only 5 regions located in the center of network in these 34 regions, in other words, very few nodes being influential in the network. In addition, it can be found that there are 19 regions whose out-degree are greater than 0,30 regions' in-degree are greater than 0 , that is, 30 regions accepted private equity investment of other regions, but only 19 regions invested in private equity in other regions. In short, most regions have the value to be invested in PE, but they do not have the ability to invest in private equity.

2) Betweenness: The betweenness of a region depicts the number of shortest paths through the region in the network, reflecting the extent to which the region is located in the middle of the other regions in the network, and measuring the region's ability to be an intermediary. A big betweenness represents that this region is in the shortest path of many region pairs, which means a lot of resources flow though this region so it can play a role in the exchange of resources between other regions, then it can acquire strong ability to control resources and other regions. Removing the region which has the biggest betweenness from the network will increase the average path length of the whole private equity investment network. The region which has the biggest betweeness is in the center of PE investment network.

TABLE II. BETWEENNESS IN PE INVESTMENT NETWORK

\begin{tabular}{|c|c|c|c|c|r|}
\hline $\begin{array}{c}\text { Orde } \\
\text { r }\end{array}$ & Region & $\begin{array}{c}\text { Between- } \\
\text { ness }\end{array}$ & Order & Region & $\begin{array}{c}\text { Betwee } \\
\text { n-ness }\end{array}$ \\
\hline 1 & Guangdong & 136.668 & 9 & Hubei & 3.307 \\
\hline 2 & Beijing & 107.976 & 10 & Sichuan & 2.51 \\
\hline 3 & Shanghai & 78.405 & 11 & Fujian & 2.067 \\
\hline 4 & Jiangsu & 46.216 & 12 & Xinjiang & 1.3 \\
\hline 5 & Chongqing & 27.593 & 13 & Hunan & 0.51 \\
\hline 6 & Zhejiang & 20.374 & 14 & Shandong & 0.367 \\
\hline 7 & Tianjin & 6.183 & 15 & Jiangxi & 0.2 \\
\hline 8 & Anhui & 3.326 & 16 & Guangxi & 0 \\
\hline
\end{tabular}

As can be seen from Tab. II, Guangdong has the largest betweenness, followed by Beijing and Shanghai, these areas have strong resource control capacity; there are 19 regions whose betweenness is 0 in the whole 34 regions, they have no resource control capacity. In the whole network, less than $10 \%$ of regions have strong resources control capacity, most regions have weak resource control capacity, and even $55.9 \%$ of the regions do not have the ability to control resources. Therefore, as a whole, the ability of most China's regions to control resources is relatively weak.

3) Closeness centrality: The closeness centrality of a node measures the closeness of the node to other nodes. A node has a great closeness centrality if all the shortest paths between this node and other nodes are short. The greater the closeness centrality of a node, the shorter the time it takes for the message to be passed from the node to the other, and it is more relevant to the other nodes, so that it is at the center of the network. In a cross-regional private equity investment network, the greater the closeness centrality of a region, the less it will be affected by other regions when conducting cross-regional private equity investments. Table 3 is the results of closeness centrality. 
TABLE III. LIST OF IN-CLOSENESS AND OUT-CLOSENESS IN PRIVATE EQUITY INVESTMENT NETWORK

\begin{tabular}{|c|l|r|l|r|}
\hline Order & Region & Out-closeness & \multicolumn{1}{|c|}{ Region } & In-closeness \\
\hline 1 & Beijing & 27.50 & Guangdong & 16.50 \\
\hline 2 & Guangdong & 26.50 & Beijing & 15.00 \\
\hline 3 & Shanghai & 25.00 & Shanghai & 14.83 \\
\hline 4 & Jiangsu & 22.00 & Henan & 14.33 \\
\hline 5 & Zhejiang & 22.00 & Jiangsu & 14.00 \\
\hline 6 & Tianjin & 21.00 & Shandong & 13.83 \\
\hline 7 & Tibet & 20.50 & Zhejiang & 13.50 \\
\hline 8 & Xinjiang & 19.00 & Chongqing & 13.00 \\
\hline 9 & Fujian & 18.83 & Hubei & 12.50 \\
\hline 10 & Hubei & 18.00 & Shaanxi & 12.50 \\
\hline
\end{tabular}

As can be seen from the results in Tab. III, Beijing, Shanghai and Guangdong both have a large in-closeness and out-closeness, indicating that the three regions are at the center in the entire network. Northeast, northwest, southwest have small in-closeness and out-closeness, they are at the periphery of the network (only the top 10 regions are listed).

4) Comparison of three indicators: Comparing the results of these three indicators, it can be found that the results of the degree analysis, betweenness analysis and the closeness centrality analysis are consistent; the region which has large degree always has large betweenness and closeness. These regions participated in the private equity investment activities more frequently, and the investment amount is larger, too. These three indicators of Guangdong, Shanghai, Jiangsu and Zhejiang are in the forefront, indicating that the five regions are in the center of the network, the other areas are in the edge of the network. There are only 5 regions in the center, and comparing with the regions at the edge of the network, the huge difference indicates that the development of China's PE investment is not uniform in different regions.

\section{Core-periphery Analysis}

Based on the tightness of the links between regions in the private equity investment network, we divided these regions into two parts, core regions and periphery regions. Regions in the core areas occupy a more important position. After calculation, Jiangsu, Fujian, Beijing, Guangdong and Shanghai belong to core regions; the other regions belong to periphery regions. From 2012 to 2016, the total cross-regional private equity investment amount was 4149108 million, while the cross-regional private equity investment amount among the five core regions was 97112.11 million, accounting for $42.72 \%$ of the total, indicating that the activity and intensity of the core regional enterprises participating in private equity investment is much higher than that of non-core regional enterprises.

The network density of the core regional network is 0.9 [9/ $(4 * 5)]$, and the network density of the non-core regional network is only $0.0530[43 /(298 * 29)]$, which means the link between the core regions is very close, almost forming a fully interoperable network, while the link between non-core regions is very weak since the network is very sparse. In addition, the link density between the non-core region and the core region is $0.1007[113 /(33 * 34)]$, is higher than that of the non-core area network-0.0530, indicating that the non-core regional enterprises are more likely to carry out mutual private equity investment with core regional enterprises. Specifically, non- core regional enterprises are more likely to be invested in private equity by core regional enterprises.

\section{CONCLUSION}

This paper uses the social network analysis method to build a complex network model, analyzes the structure of the network and its economic significance, and obtains the following main conclusions:

The development of private equity investment in various regions of China is very uneven. Few regions have a great degree, betweenness and closeness, they are in a dominant position in the network, and the investment frequency and investment amount of them are much higher than that of other regions. In fact, the private equity investment amount investors and financials both are in the core region is 4525992 million Yuan from 2012 to 2016, accounting for $46.73 \%$ of the total; the private equity investment amount whose investors or financials are in the core region is 8954398 million Yuan from 2012 to 2016 , accounting for $92.46 \%$ of the total, which can also explain the uneven development of China's private equity investment. These advantage regions are concentrated in Beijing and China's southeastern coastal provinces and cities, of which Beijing is China's political center, which gathered a large number of talent and capital; the southeast coastal zone is economically developed, not only having abundant funds but also providing good development opportunities to entrepreneurs, so they choose to create companies in these regions, which provides a broad choice for investment institution in turn.

Enterprises both in the core and non-core regions tend to invest in private equity in or be invested in private equity by enterprises in the core region. The density of the core regional network is 0.9 , which is much higher than that of the overall network -0.1551 , indicating that the core regional enterprises are much more attractive to each other than non-core regional enterprises' attraction to the core regional enterprises; the link density between the non-core region and the core region is 0.1007 , which is higher than the density of the non-core regional network - 0.0530, indicating that the non-core regional enterprises are less attractive to each other than core regional enterprises' attraction to the non-core regional enterprises. Core regions always have policy, economic and other advantages. For example, since the SFC is located in Beijing, so the investment institutions in Beijing are easier to establish a good contact with the SFC, then they are more familiar with the approval process, and easier to grasp the timing of successful listing. This advantage is unique, it is attractive to other regions' start-up enterprises, so they are willing to cooperate with Beijing's investment institutions.

\section{ACKNOWLEDGEMENT}

This paper is partially supported by the Fundamental Research Funds for the Central Universities (project grant number: 2013JBM036).

\section{REFERENCES}

[1] Albert R. and BarabásiA-L., 2002, Statistical mechanics of complex network[J] .Review of Modern Physics, 74. 
[2] BarabásiA-L. and Albert R., 1999, Emergence of scaling in random networks[J].Science, 286.

[3] Ming Zheng, Shi Chen.Study on the Measurement of Investment Relationship of Private Equity Funds [J]. Securities Market Herald, 2011, (10): 43-49. 